\title{
NNLO QCD corrections to diphoton production with an additional jet at the LHC
}

\author{
Herschel A. Chawdhry, ${ }^{a}$ Michał Czakon, ${ }^{b}$ Alexander Mitov ${ }^{c}$ and Rene Poncelet ${ }^{c}$ \\ ${ }^{a}$ Rudolf Peierls Centre for Theoretical Physics, Clarendon Laboratory, University of Oxford, \\ Oxford OX1 3PU, United Kingdom \\ ${ }^{b}$ Institut für Theoretische Teilchenphysik und Kosmologie, RWTH Aachen University, \\ D-52056 Aachen, Germany \\ ${ }^{c}$ Cavendish Laboratory, University of Cambridge, \\ Cambridge CВ3 OHE, United Kingdom \\ E-mail: herschel.chawdhry@physics.ox.ac.uk, \\ mczakon@physik.rwth-aachen.de, adm74@cam.ac.uk, \\ poncelet@hep.phy.cam.ac.uk
}

ABSTRACT: We calculate the NNLO QCD corrections to diphoton production with an additional jet at the LHC. Our calculation represents the first NNLO-accurate prediction for the transverse momentum distribution of the diphoton system. The improvement in the accuracy of the theoretical prediction is significant, by a factor of up to four relative to NLO QCD as estimated through scale variations. Our calculation is exact except for the finite remainder of the two-loop amplitude which is included at leading color. The numerical impact of this approximated contribution is small. The results of this work are expected to further our understanding of the Higgs boson sector and of the behavior of higher-order corrections to LHC processes.

KEYWORDS: QCD Phenomenology

ArXiv EPrint: 2105.06940 


\section{Contents}

1 Introduction $r$

2 Setup of the calculation $r$

3 Phenomenological results $\quad 3$

4 Conclusions $\quad 7$

\section{Introduction}

The production of a pair of photons at the LHC is of special interest. On the one hand this process represents the main background to the cleanest Higgs boson decay channel $h \rightarrow \gamma \gamma$. On the other hand it is a process where our ability to accurately predict LHC cross sections by including higher-order QCD corrections can reliably be tested.

Inclusive diphoton production $p p \rightarrow \gamma \gamma+X$ has been studied extensively at NNLO in QCD [1-5]. Prior work also includes NLO QCD corrections [6] or electroweak effects [7], as well as photon isolation [8]. Beyond NLO, resummation effects have been included at least to NNLL [9-12]. Interference between $\gamma \gamma+$ jet and $h \rightarrow \gamma \gamma+j$ have also been investigated [13]. A detailed analysis of the limitations of existing results can be found in ref. [14].

A distinguishing feature of this process is the presence of very large higher-order QCD corrections which has raised some questions about the reliability of such higher-order predictions for this process. With time our understanding of the behavior of higher-order corrections in this case has developed significantly. It is presently believed [3] that starting with $\mathrm{N}^{3} \mathrm{LO}$, perturbative corrections will be much more mild and consistent with perturbative convergence. A similar conclusion has also been reached recently for the process $p p \rightarrow \gamma \gamma \gamma[15,16]$. Clearly it is very desirable to have a full $\mathrm{N}^{3} \mathrm{LO}$ accurate calculation of inclusive diphoton production where these ideas can be tested and hopefully validated. The present work, together with the recently computed 3-loop amplitudes for diphoton production at the LHC [17], represents a significant step in this direction.

The transverse momentum of the photon pair, $p_{T}(\gamma \gamma)$, plays a special role in inclusive diphoton production $p p \rightarrow \gamma \gamma+X$. As is well known, due to the fact that at leading order $p_{T}(\gamma \gamma)=0$, an NNLO-accurate calculation of inclusive diphoton production is only NLO-accurate for the $p_{T}(\gamma \gamma)$ distribution. To achieve NNLO accuracy for $p_{T}(\gamma \gamma)$ at nonzero $p_{T}(\gamma \gamma)$ one needs to compute the NNLO QCD corrections for the process $p p \rightarrow \gamma \gamma+$ jet $+X$. The present work presents the first calculation of $p p \rightarrow \gamma \gamma+$ jet $+X$ in NNLO QCD and makes public NNLO QCD predictions for a number of diphoton observables at nonzero $p_{T}(\gamma \gamma)$. 
The main reason the $p_{T}$ distribution of the diphoton system is of special interest is that it represents the main background for Higgs production at high $p_{T}$. High- $p_{T}$ Higgs production is relevant for Dark Matter searches [18, 19] and for disentangling the nature of the Higgs boson's local vertex [20] which is not possible at low $p_{T}$ where the effective $g g h$ vertex describes Higgs production well. The $p_{T}(\gamma \gamma)$ distribution, together with the angular distribution of the two photons in the Collins-Soper frame [21], represents a strong discriminator for the spin of a possible resonance decaying to two photons [22]. For further details about high- $p_{T}$ Higgs production we refer the reader to the recent review [23].

This work is organized as follows: in section 2 we briefly describe our calculation while in section 3 we present our predictions for a number of differential distributions. Our conclusions are given in section 4 .

\section{Setup of the calculation}

The calculation is performed in the STRIPPER approach [24-26]. The approach has already been applied in the calculation of NNLO QCD corrections to top-pair [27-31], inclusive jet [32], three-photon [15], $W+c$ jet [33], identified $B$-hadron [34] and polarized $W$-pair [35] production at the LHC. A detailed description of the technical aspects of our implementation has been given in ref. [32].

All tree-level diagrams are computed with the avhlib library [36, 37]. The contributing one-loop amplitudes, including the loop-induced contribution, are obtained from the library OpenLoops [38, 39].

The relevant two-loop contributions $q \bar{q} \rightarrow g \gamma \gamma$ and $q g \rightarrow q \gamma \gamma$ are handled in the following way. We first separate the finite remainders $\mathcal{H}^{(2)}\left(\mu_{R}^{2}\right)$ of the two-loop amplitudes from their infrared poles. The latter can be predicted exactly and we have included them, including their finite contributions, without any approximation. In terms of the scale dependence of the two-loop finite remainder $\mathcal{H}^{(2)}$ (defined as in ref. [26]):

$$
\mathcal{H}^{(2)}\left(\mu_{R}^{2}\right)=\mathcal{H}^{(2)}\left(s_{12}\right)+\sum_{i=1}^{4} c_{i} \ln ^{i}\left(\frac{\mu_{R}^{2}}{s_{12}}\right),
$$

where $s_{12}$ is the squared partonic center-of-mass energy, we have included without any approximation all two-loop terms $c_{i}$ corresponding to $\ln ^{i}\left(\mu_{R}^{2}\right)$ with $i \geq 1$. The scaleindependent part $\mathcal{H}^{(2)}\left(s_{12}\right)$ is included in the leading color approximation as derived in ref. [40] with the help of refs. [41-43] (an equivalent expression for the spin-averaged twoloop squared amplitude has also been derived in ref. [44]), i.e. we approximate $\mathcal{H}^{(2)}\left(s_{12}\right) \approx$ $\mathcal{H}_{\text {l.c. }}^{(2)}\left(s_{12}\right)$. This is the only approximation made in this paper. Further details about the implementation of the leading color approximation of the two-loop finite remainder can be found in ref. [15].

As a justification of the leading color approximation just described we have verified that the numerical contribution of the scale-independent part of the leading color two-loop finite remainder $\mathcal{H}_{\text {l.c. }}^{(2)}\left(s_{12}\right)$ is small. For all distributions computed here it is about $1-2 \%$ of the complete NNLO prediction in all bins. This makes it smaller than the Monte Carlo 
integration error in the differential distributions. This should serve as a concervative error estimate on the missing sub-leading color terms. More details can be found in section 3 .

We have also included the so-called loop-induced (LI) contribution $g g \rightarrow g \gamma \gamma$ which begins to contribute starting at NNLO. As we explain in section 3 its effect is about five percent and is strongly dependent on the distribution.

The QED coupling is taken to be $\alpha=1 / 137$. The strong coupling constant and parton distributions are renormalized such that they evolve with $n_{f}=5$ active flavors. Diagrams with top-quark loops are included in all contributions except the two-loop finite remainder and the one-loop squared contribution. The current calculation uses the NNPDF3.1 pdf set [45] of order that matches the order of the perturbative calculation. The value of the strong coupling constant is taken from the LHAPDF interface [46]. The central values of the factorization and renormalization scales have been fixed to:

$$
\mu_{F}^{2}=\mu_{R}^{2}=\frac{1}{4}\left(m^{2}(\gamma \gamma)+p_{T}(\gamma \gamma)^{2}\right) .
$$

This scale choice has been motivated by the scales used in refs. $[6,8]$ : we have replaced the $p_{T}$ of the jet with that of the photon pair since in our setup, see below, we have no explicit jet requirements. At LO the two scales are equivalent.

Scale uncertainty has been estimated with the help of a 7-point restricted independent variation by a factor of 2 of the scales $\mu_{F}$ and $\mu_{R}$. Since in this work we are primarily concerned with perturbative convergence and estimates of missing higher-order corrections, we have not included pdf error estimates. We expect that those are not dominant over the scale variation in the kinematic ranges considered here. We hope to include them in a future update of the present work.

Our calculation has been performed for the LHC at $13 \mathrm{TeV}$ and is subject to the following set of selection cuts: ${ }^{1}$ we require two photons satisfying the following criteria

- $p_{T}\left(\gamma_{1}\right)>30 \mathrm{GeV}, p_{T}\left(\gamma_{2}\right)>18 \mathrm{GeV}$ and $|\eta(\gamma)|<2.4$,

- Smooth photon isolation [48] with $\Delta R_{0}=0.4$ and $E_{T}^{\max }=10 \mathrm{GeV}$ (see ref. [15] for details),

- $m_{\gamma \gamma} \geq 90 \mathrm{GeV}$,

- $\Delta R(\gamma, \gamma)>0.4$,

- $p_{T}(\gamma \gamma)>20 \mathrm{GeV}$ (for lower values resummation effects become important [12]).

No additional jet requirements are imposed. In particular, infrared safety is ensured by the $p_{T}(\gamma \gamma)$ cut specified above.

\section{Phenomenological results}

In this work we calculate the NNLO QCD corrections to a number of one-dimensional distributions in the following variables: the transverse momentum of the photon pair

\footnotetext{
${ }^{1}$ This set of cuts is based on typical selection requirements, see refs. [22, 47].
} 

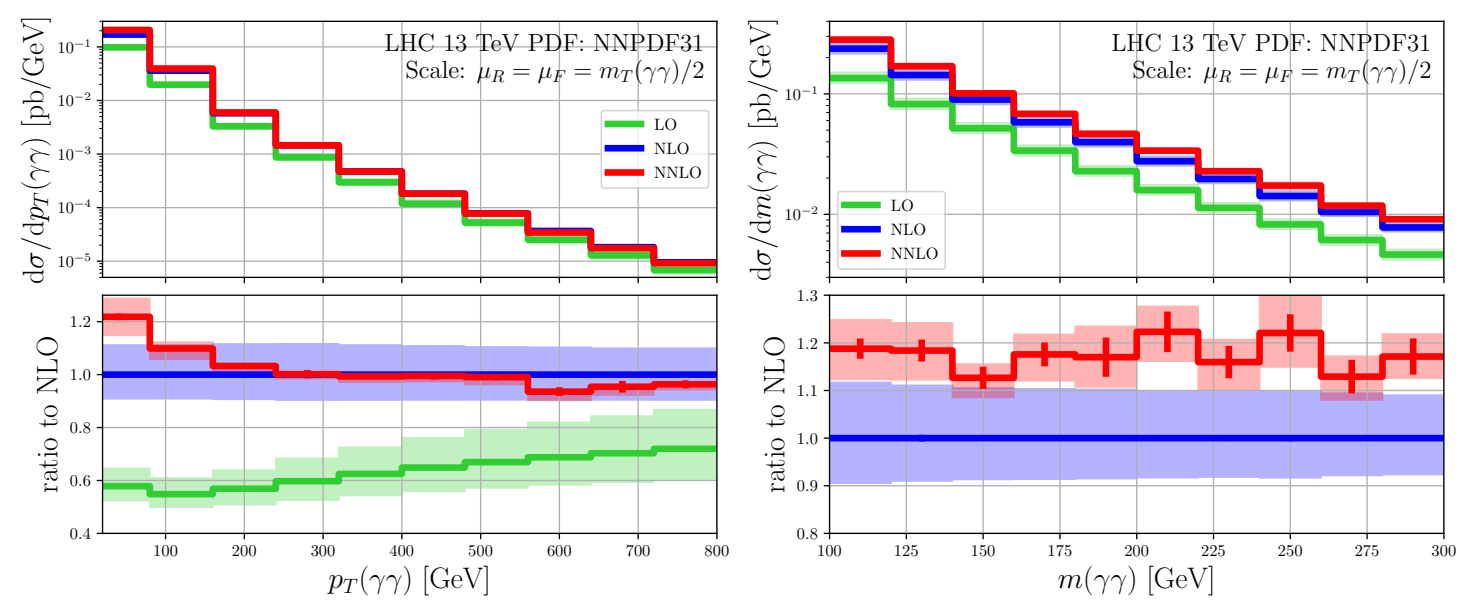

Figure 1. Absolute $p_{T}(\gamma \gamma)$ (left) and $m(\gamma \gamma)$ (right) differential distributions. Shown are the predictions in LO (green), NLO (blue), NNLO (red) QCD. The colored bands around the central scales are from 7-point scale variation. The colored bars shows the estimated Monte Carlo integration error in each bin. The lower panel shows the same distributions but relative to the NLO central scale prediction.
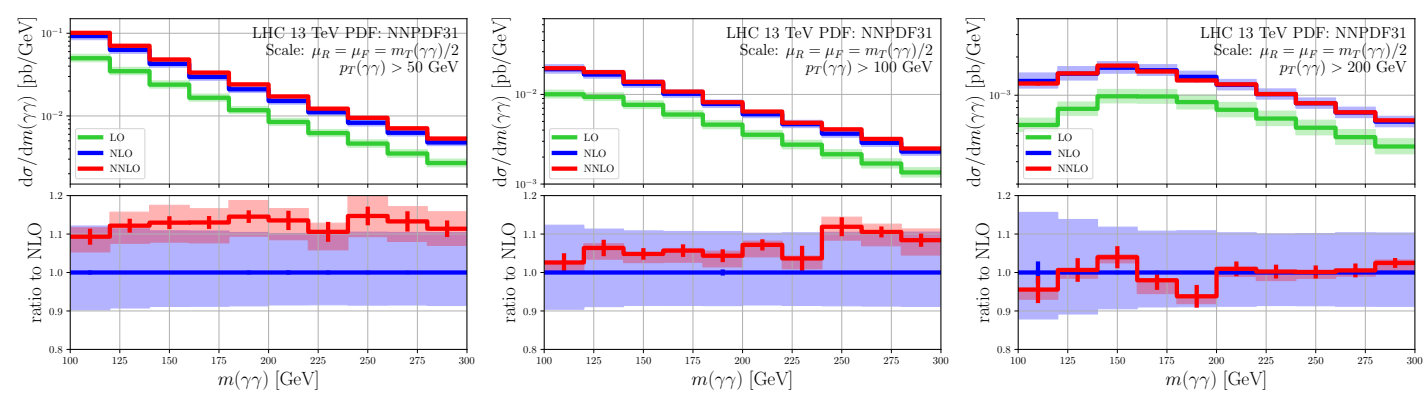

Figure 2. As in figure 1 but for the $m(\gamma \gamma)$ distribution subjected to different $p_{T}(\gamma \gamma)$ cuts: $p_{T}(\gamma \gamma)>$ $50 \mathrm{GeV}$ (left), $p_{T}(\gamma \gamma)>100 \mathrm{GeV}$ (center) and $p_{T}(\gamma \gamma)>200 \mathrm{GeV}$ (right).

$p_{T}(\gamma \gamma)$, the invariant mass of the two photons $m(\gamma \gamma)$, the angle between the two photons in the Collins-Soper frame $\phi_{C S}$, the absolute difference in rapidities of the two photons $\Delta y(\gamma \gamma)=\left|y\left(\gamma_{1}\right)-y\left(\gamma_{2}\right)\right|$, the azimuthal angle between the two photons $\Delta \phi(\gamma \gamma)$ and the absolute rapidity of the photon pair $|y(\gamma \gamma)|$. We also calculate the NNLO QCD corrections to the following two-dimensional distributions: $m(\gamma \gamma) \otimes p_{T}(\gamma \gamma)$ and $\phi_{C S} \otimes m(\gamma \gamma)$.

We first discuss the $p_{T}(\gamma \gamma)$ differential distribution which is of central interest to this work. The distribution is shown in figure 1. As can be seen from this figure, the NLO QCD correction is very significant relative to the LO one. In particular, the scale uncertainty bands at LO and NLO do not overlap anywhere. This behavior is easy to understand based on the properties of inclusive diphoton production through NNLO. Clearly, a reliable prediction of this observable requires the inclusion of, at least, the NNLO QCD corrections.

As can be seen from figure 1 the inclusion of the NNLO corrections has a major stabilizing impact on the $p_{T}(\gamma \gamma)$ distribution. With the exception of the very low $p_{T}(\gamma \gamma)$ region which we will discuss shortly, the scale uncertainty decreases significantly, by a factor 
of about four, relative to NLO. Moreover the NLO and NNLO scale bands now overlap everywhere. Such a behavior is consistent with the expected stabilization of the inclusive diphoton production cross-section starting at $\mathrm{N}^{3} \mathrm{LO}$.

The low- $p_{T}(\gamma \gamma)$ behavior of this distribution deserves special attention. A fixed-order perturbative description would not be adequate for $p_{T}(\gamma \gamma)$ below about $20 \mathrm{GeV}$ due to the importance of resummation effects, but we expect it to be reliable for larger $p_{T}(\gamma \gamma)$ values. For this reason one may wonder why the low- $p_{T}(\gamma \gamma)$ part of the spectrum shows significantly increased scale dependence and larger NNLO/NLO $K$-factor. This behavior may be influenced by resummation effects however we do not expect them to be the dominant ones. We suspect that the main factor behind it is the loop-induced contribution $g g \rightarrow g \gamma \gamma$ which only starts to contribute at NNLO.

The impact of this contribution is shown in figure 6 as a ratio of the full NNLO to the NNLO excluding this contribution. As figure 6 indicates the loop-induced correction is concentrated at relatively low $p_{T}(\gamma \gamma)$ values and becomes completely negligible for $p_{T}(\gamma \gamma)$ values about $200 \mathrm{GeV}$ or larger. If the loop induced correction is excluded, the scale dependence of the first bin becomes smaller by about a factor of two and the NNLO/NLO $K$-factor also decreases by a factor of about two.

Our findings indicate that at the level of NNLO QCD corrections, the loop-induced contribution becomes significant. This contribution can be tamed further, by including the NLO QCD correction to the loop-induced contribution (which is a partial $\mathrm{N}^{3} \mathrm{LO}$ contribution for this process). Such a calculation requires the two-loop amplitude for the process $g g \rightarrow g \gamma \gamma$. This result is not available in the literature but it is certainly within reach given the number of other five-point two-loop QCD amplitudes that have been computed.

Overall, the scale uncertainty of the $p_{T}(\gamma \gamma)$ spectrum at NNLO is rather small about couple of percent for diphoton $p_{T}$ 's above $200 \mathrm{GeV}$ or so. This implies that this observable is well described within perturbative QCD and can be used in precision analyses of Higgs physics and searches for resonances decaying to diphoton final states. The dominant uncertainty at large $p_{T}(\gamma \gamma)$ is due to the Monte Carlo integration error of the calculation itself. It can be further improved albeit at a significant computational cost. A future update may also include pdf uncertainties, electroweak effects as well as the NLO correction to the loop-induced process. Finally, the effects from the leading color approximation used here may also need to be improved upon. As can be seen from figure 6 the approximated contribution is a rather small 1-2\% effect and is much smaller than the MC error. It can also be improved upon once the complete two-loop amplitudes for this process become available.

We next turn our attention to the $m(\gamma \gamma)$ distribution. It is shown in figures 1 and 2 . This distribution is significant for any search of resonances decaying to diphotons at nonzero $p_{T}$. To thoroughly understand the interplay between $m(\gamma \gamma)$ and $p_{T}(\gamma \gamma)$ we have shown the $m(\gamma \gamma)$ distribution in several ways. In figure 1 we show the $m(\gamma \gamma)$ distribution subjected only to our default selection cuts. In figure 2 we show the same distribution but with more stringent $p_{T}(\gamma \gamma)$ cuts of $50 \mathrm{GeV}, 100 \mathrm{GeV}$ and $200 \mathrm{GeV}$. A summary of the same result (only the NLO and NNLO are displayed) is shown in figure 5 (right). Figure 5 (center) shows the $m(\gamma \gamma)$ distributions for several slices of $p_{T}(\gamma \gamma)$. 

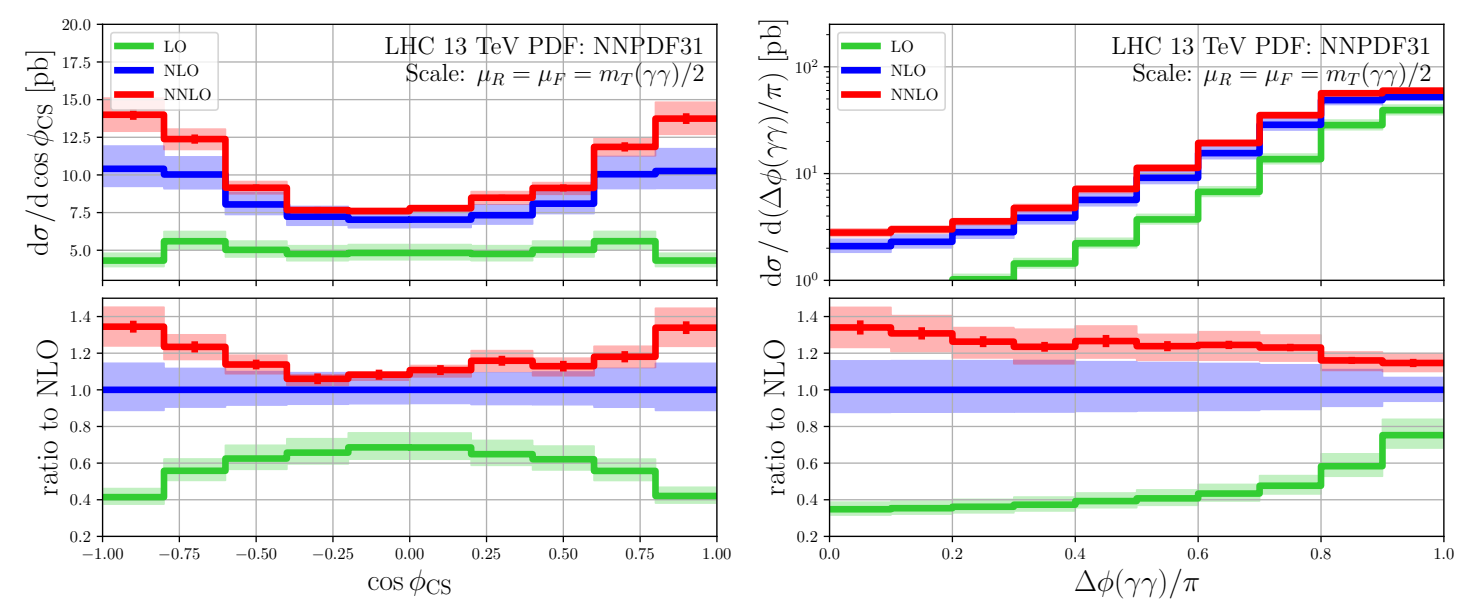

Figure 3. As in figure 1 but for the angular distributions in $\phi_{C S}$ (left) and $\Delta \phi(\gamma \gamma)$ (right).

From these plots it is clear that the $m(\gamma \gamma)$ distribution has a pattern of higherorder corrections that is similar to $p_{T}(\gamma \gamma)$ : large NLO/LO corrections and much smaller NNLO/NLO ones. The size of the NNLO corrections strongly depends on the $p_{T}(\gamma \gamma)$ cut and they decrease as the cut increases. For small values of the $p_{T}(\gamma \gamma)$ cut the NNLO and NLO scale bands do not overlap while they fully overlap for $p_{T}(\gamma \gamma)$ cuts above $100 \mathrm{GeV}$. One may wonder if such a behavior is related to the loop-induced contribution. In figure 6 we have shown its effect for all $p_{T}(\gamma \gamma)$ cuts considered in this work. From this one can conclude that indeed the size of the loop-induced correction is consistent with the nonoverlap of the NLO and NNLO scale bands. This means that for theoretical predictions to be reliable with full NNLO accuracy for $p_{T}(\gamma \gamma)$ cuts below $100 \mathrm{GeV}$ or so, the NLO corrections to the loop induced contributions might need to be included.

In general, the effect of the NNLO correction on the $m(\gamma \gamma)$ distribution is a rather flat shift with respect to the NLO one and leads to a decrease of the scale uncertainty by a factor of about two at low $p_{T}(\gamma \gamma)$ and four or more at large $p_{T}(\gamma \gamma)$. Another important source of error is the MC integration one. The effect from the leading color approximation in the finite remainder is at the percent level and therefore insignificant.

In figure 3 we show distributions in the angular variables $\phi_{C S}$ and $\Delta \phi(\gamma \gamma)$ while in figure 4 we show the $\Delta y(\gamma \gamma)$ and $|y(\gamma \gamma)|$ rapidity distributions. The $\phi_{C S}$ distribution in slices of $m(\gamma \gamma)$ is shown in figure 5 (left). All these distributions have very large NLO/LO $K$-factors. Unlike the $p_{T}(\gamma \gamma)$ and $m(\gamma \gamma)$ distributions, however, they also have sizable NNLO corrections which in most bins are outside the NLO uncertainty bands. This pattern of higher order corrections indicates that for the scale used in this work, the NLO approximation is inadequate for describing these distributions.

Based on the above observations one may question the presence of perturbative stability in these variables. As a first step towards analyzing this we consider the behavior of the NNLO prediction without the loop-induced contribution (in the following we refer to it as NNLO-minus-LI). The numerical impact of the loop-induced contribution for each differential distribution can be seen in figure 6 and figure 7 . We observe the following. 

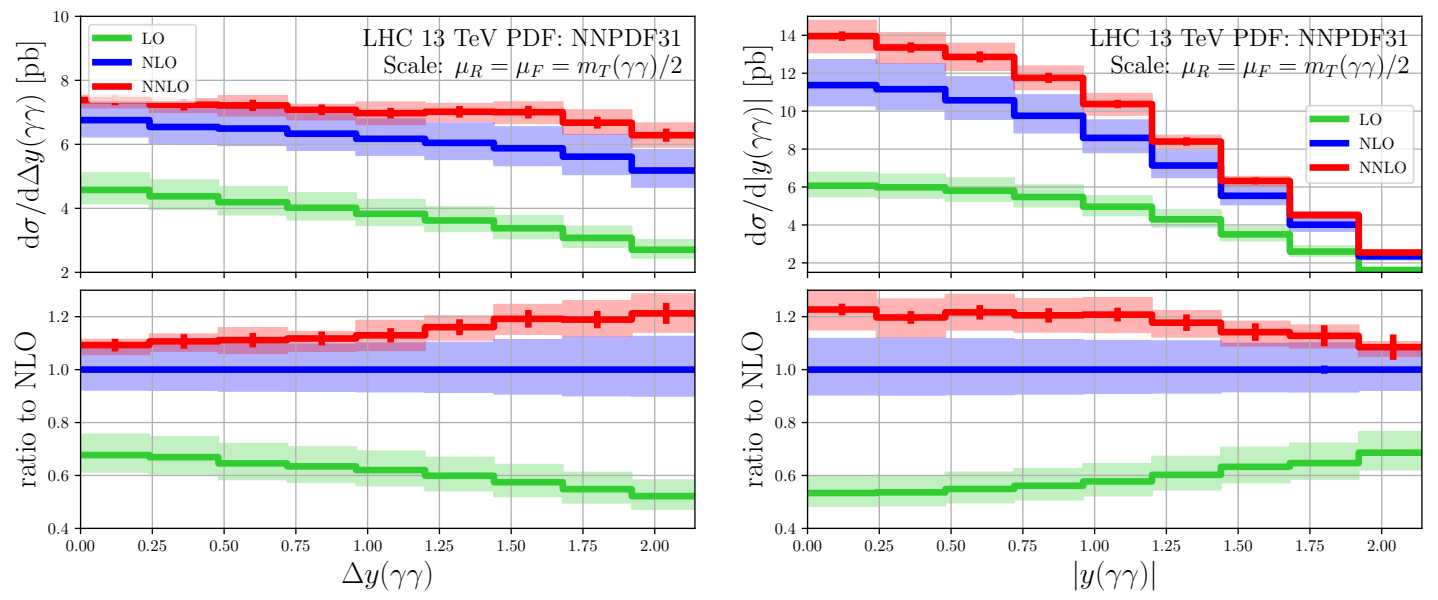

Figure 4. As in figure 1 but for the following rapidity distributions: $\Delta y(\gamma \gamma)($ left $)$ and $|y(\gamma \gamma)|$ (right).

For the $\phi_{C S}$ distribution the NNLO-minus-LI scale uncertainty band is mostly within the NLO one or the two bands overlap. This is not the case for the first and last bins of this distribution, however, the behavior of the $\phi_{C S}$ distribution in these two bins is strongly affected by the kinematic cuts. The NNLO-minus-LI band for the $\Delta y(\gamma \gamma)$ distribution overlaps in all bins with the NLO one. Same can be observed for the case of the $|y(\gamma \gamma)|$ distribution. In fact, the only distribution for which the NNLO-minus-LI and NLO scale uncertainty bands do not mostly overlap is the $\Delta \phi(\gamma \gamma)$ one. For this distribution we observe that the NNLO-minus-LI and NLO scale uncertainty bands overlap for $\Delta \phi(\gamma \gamma) / \pi>0.6$ while below this value they are not very far apart, see figure 7. Given that the NLO/LO $K$-factor in this region is more than a factor of two it seems that such a non-overlap is not too concerning.

From the above discussion it seems reasonable to conclude that the non-overlap between NNLO and NLO scale uncertainty bands observed in the angular and rapidity diphoton distributions is somewhat affected by the loop-induced contribution. It is therefore plausible to assume that the inclusion of this contribution's NLO correction may alleviate this non-overlap. Other factors that may be affecting this behavior is the choice of scale as well as resummation effects which are relevant at low $p_{T}(\gamma \gamma)$. A detailed investigation of those is however outside the scope of this work. On the other hand, as can also be seen from figure 6, the two-loop finite remainder has a rather small contribution and, therefore, we do not expect these distributions to be significantly affected by two-loop subleading color corrections.

\section{Conclusions}

In this work we calculate the NNLO QCD corrections to the process $p p \rightarrow \gamma \gamma+$ jet. This process is the main background to high- $p_{T}$ Higgs boson production decaying to two photons. The main result of this work is the calculation of the diphoton $p_{T}$ spectrum with NNLO accuracy. The NNLO correction to this variable is important and it brings 

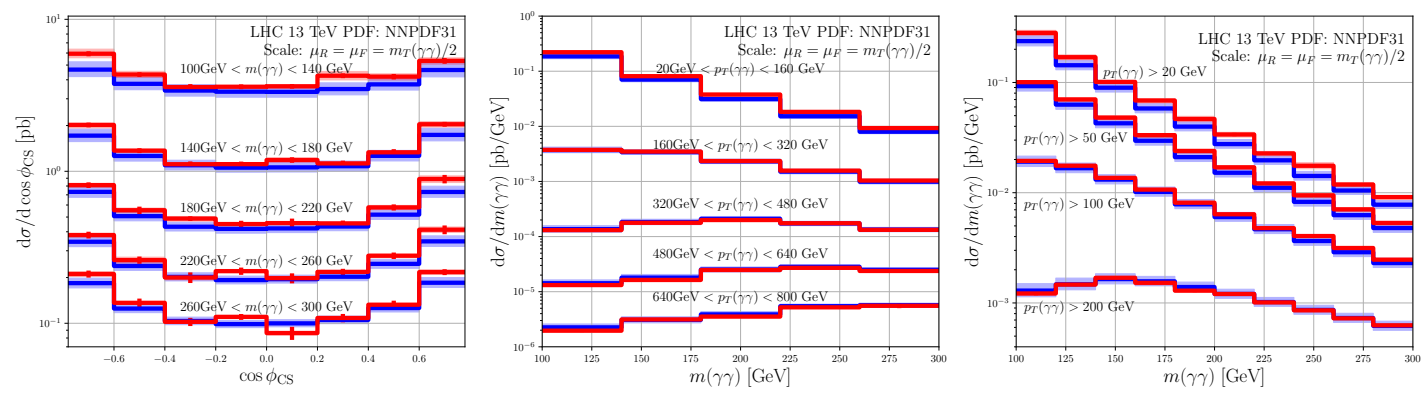

Figure 5. Two-dimensional differential distributions: in $\phi_{C S} \otimes m(\gamma \gamma)$ (left) and in $m(\gamma \gamma) \otimes p_{T}(\gamma \gamma)$ but shown in two alternative forms and for different choice of bins: in $p_{T}(\gamma \gamma)$ bins (center) and with $p_{T}(\gamma \gamma)$ cuts (right). Only the NLO and NNLO central scale predictions and scale variation bands are shown. Note that the figure to the right shows the same results that already appear in figures 1,2 .

the uncertainty from un-calculated higher-order corrections down to a couple of percent at intermediate and large values of $p_{T}(\gamma \gamma)$. Overall, the quality of the theoretical prediction for this distribution is very high and it appears to be under good theoretical control. The same conclusion applies for double differential distributions in $p_{T}(\gamma \gamma)$ and $m(\gamma \gamma)$.

We have suggested various possible avenues for further improving the quality of the theoretical predictions in this process. They include the calculations of the partial $\mathrm{N}^{3} \mathrm{LO}$ corrections due to loop induced processes which can be calculated with the help of NLO technology. The only missing ingredient for such a calculation is the two-loop amplitude for the process $g g \rightarrow g \gamma \gamma$ whose calculation is within reach. A more extensive study of possible scale choices for this process might also be beneficial given the very high precision reached in the $p_{T}(\gamma \gamma)$ distribution. Merging our fixed-order calculations with resummed calculations will allow for a quality description of the $p_{T}(\gamma \gamma)$ spectrum from very high down to very low values of $p_{T}(\gamma \gamma)$.

We conclude by stressing that the quality of the theoretical description achieved for this process is high which makes it possible to use it in background estimates for Higgs boson studies and related searches as well as in dedicated measurements of diphoton production.

Note added. After the completion of the current work, ref. [49] appeared. It provides the subleading-colour expressions for the two-loop amplitudes for this process. We will include them in a future update of this work.

\section{Acknowledgments}

The work of M.C. was supported by the Deutsche Forschungsgemeinschaft under grant 396021762 - TRR 257. The research of A.M. and R.P. has received funding from the European Research Council (ERC) under the European Union's Horizon 2020 Research and Innovation Programme (grant agreement no. 683211). A.M. was also supported by the UK STFC grants ST/L002760/1 and ST/K004883/1. The research of H.C. is supported by the ERC Starting Grant 804394 HipQCD. A.M. acknowledges the use of the DiRAC Cumulus HPC facility under Grant No. PPSP226. 

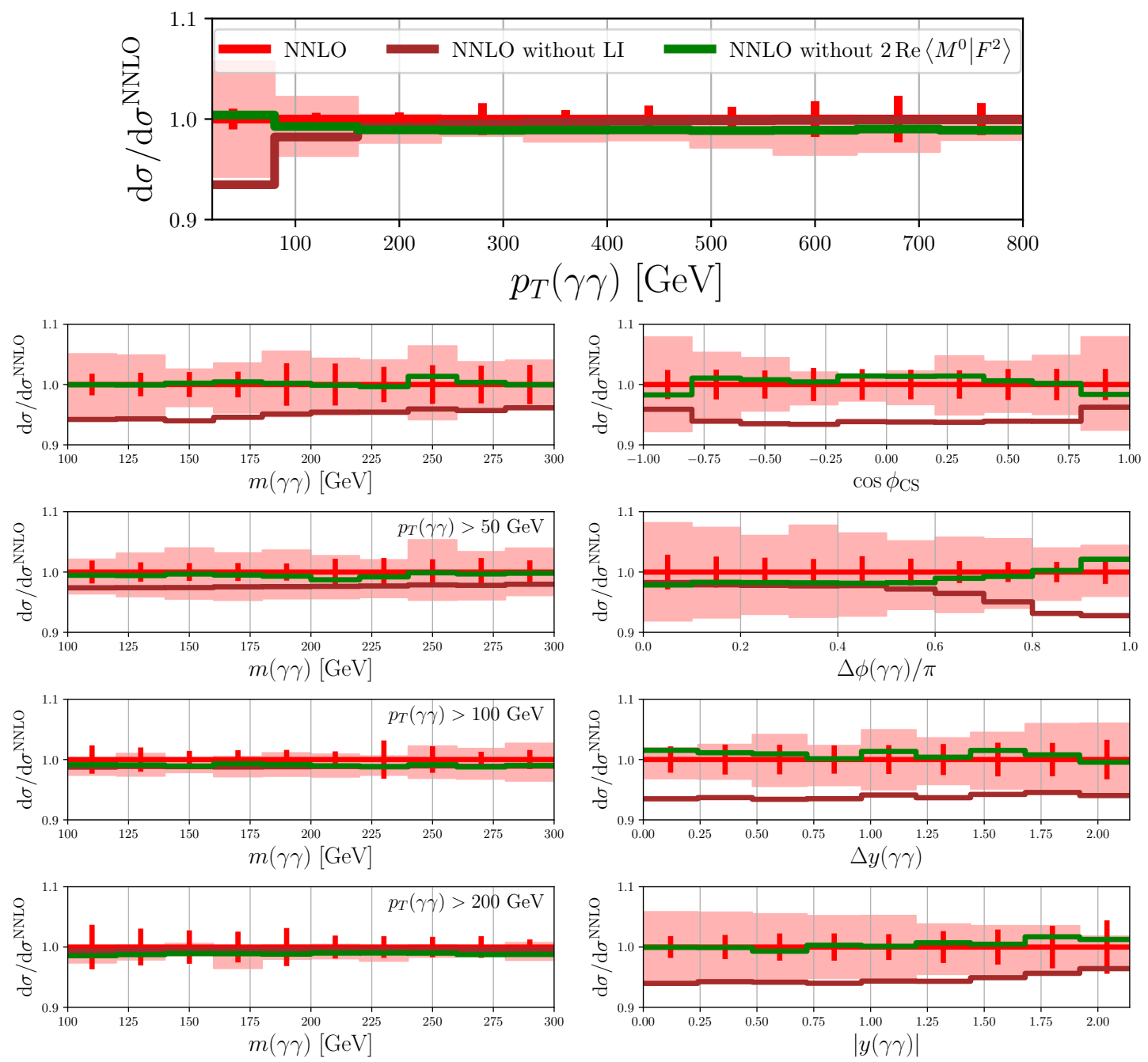

Figure 6. Comparison of various approximations to the NNLO differential predictions: the complete NNLO prediction (red), NNLO excluding the loop-induced contribution (dark brown) and NNLO excluding the finite remainder $\mathcal{H}^{(2)}\left(s_{12}\right)$ defined in eq. (2.1) (green). The colored bars shows the MC integration error of the complete NNLO prediction while the red band shows its scale variation. 

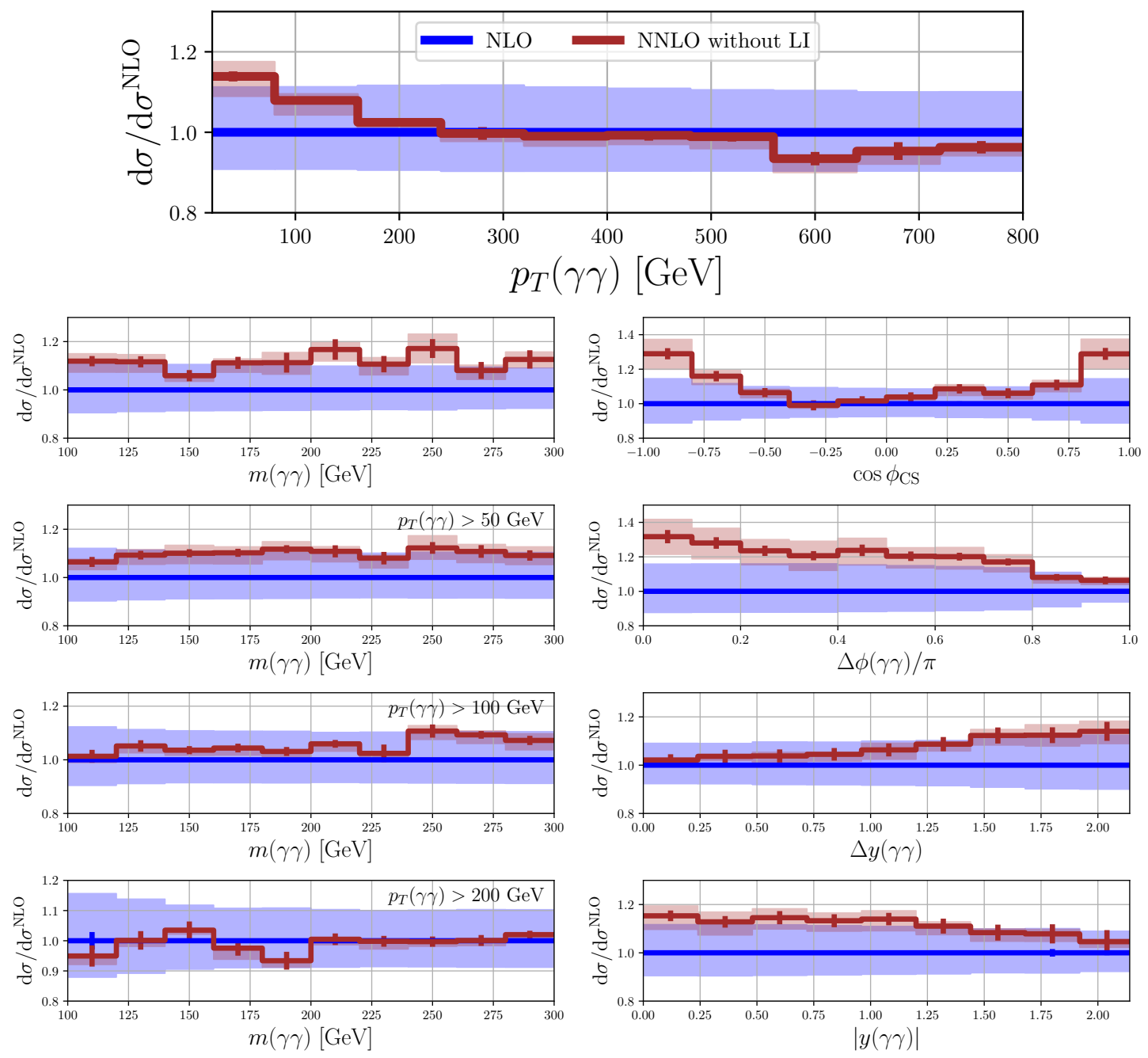

Figure 7. Comparison of NLO (blue) scale bands to NNLO QCD excluding the loop-induced contribution (dark brown). 
Open Access. This article is distributed under the terms of the Creative Commons Attribution License (CC-BY 4.0), which permits any use, distribution and reproduction in any medium, provided the original author(s) and source are credited.

\section{References}

[1] S. Catani, L. Cieri, D. de Florian, G. Ferrera and M. Grazzini, Diphoton production at hadron colliders: a fully-differential QCD calculation at NNLO, Phys. Rev. Lett. 108 (2012) 072001 [Erratum ibid. 117 (2016) 089901] [arXiv: 1110.2375] [INSPIRE].

[2] J.M. Campbell, R.K. Ellis, Y. Li and C. Williams, Predictions for diphoton production at the LHC through NNLO in QCD, JHEP 07 (2016) 148 [arXiv:1603.02663] [INSPIRE].

[3] S. Catani, L. Cieri, D. de Florian, G. Ferrera and M. Grazzini, Diphoton production at the LHC: a QCD study up to NNLO, JHEP 04 (2018) 142 [arXiv: 1802.02095] [INSPIRE].

[4] T. Gehrmann, N. Glover, A. Huss and J. Whitehead, Scale and isolation sensitivity of diphoton distributions at the LHC, JHEP 01 (2021) 108 [arXiv: 2009.11310] [INSPIRE].

[5] S. Alioli et al., Precise predictions for photon pair production matched to parton showers in GENEVA, JHEP 04 (2021) 041 [arXiv: 2010.10498] [inSPIRE].

[6] V. Del Duca, F. Maltoni, Z. Nagy and Z. Trócsányi, QCD radiative corrections to prompt diphoton production in association with a jet at hadron colliders, JHEP 04 (2003) 059 [hep-ph/0303012] [INSPIRE].

[7] M. Chiesa, N. Greiner, M. Schönherr and F. Tramontano, Electroweak corrections to diphoton plus jets, JHEP 10 (2017) 181 [arXiv:1706.09022] [INSPIRE].

[8] T. Gehrmann, N. Greiner and G. Heinrich, Photon isolation effects at NLO in $\gamma \gamma+$ jet final states in hadronic collisions, JHEP 06 (2013) 058 [Erratum ibid. 06 (2014) 076] [arXiv: 1303.0824] [INSPIRE].

[9] L. Cieri, F. Coradeschi and D. de Florian, Diphoton production at hadron colliders: transverse-momentum resummation at next-to-next-to-leading logarithmic accuracy, JHEP 06 (2015) 185 [arXiv: 1505. 03162] [INSPIRE].

[10] F. Coradeschi and T. Cridge, reSolve - A transverse momentum resummation tool, Comput. Phys. Commun. 238 (2019) 262 [arXiv:1711. 02083] [inSPIRE].

[11] M.A. Ebert and F.J. Tackmann, Resummation of Transverse Momentum Distributions in Distribution Space, JHEP 02 (2017) 110 [arXiv:1611.08610] [INSPIRE].

[12] T. Becher and T. Neumann, Fiducial $q_{T}$ resummation of color-singlet processes at $N^{3} L L+N N L O, J H E P 03$ (2021) 199 [arXiv: 2009.11437] [INSPIRE].

[13] L. Cieri, F. Coradeschi, D. de Florian and N. Fidanza, Transverse-momentum resummation for the signal-background interference in the $H \rightarrow \gamma \gamma$ channel at the LHC, Phys. Rev. D 96 (2017) 054003 [arXiv: 1706. 07331] [INSPIRE].

[14] S. Amoroso et al., Les Houches 2019: Physics at TeV Colliders: Standard Model Working Group Report, in 11th Les Houches Workshop on Physics at TeV Colliders: PhysTeV Les Houches, (2020) [arXiv:2003.01700] [INSPIRE].

[15] H.A. Chawdhry, M.L. Czakon, A. Mitov and R. Poncelet, NNLO QCD corrections to three-photon production at the LHC, JHEP 02 (2020) 057 [arXiv: 1911.00479] [INSPIRE]. 
[16] S. Kallweit, V. Sotnikov and M. Wiesemann, Triphoton production at hadron colliders in NNLO QCD, Phys. Lett. B 812 (2021) 136013 [arXiv:2010.04681] [InSPIRE].

[17] F. Caola, A. Von Manteuffel and L. Tancredi, Diphoton Amplitudes in Three-Loop Quantum Chromodynamics, Phys. Rev. Lett. 126 (2021) 112004 [arXiv:2011.13946] [INSPIRE].

[18] CMS collaboration, Search for dark matter produced in association with a Higgs boson decaying to $\gamma \gamma$ or $\tau^{+} \tau^{-}$at $\sqrt{s}=13 \mathrm{TeV}$, JHEP 09 (2018) 046 [arXiv:1806.04771] [INSPIRE].

[19] ATLAS collaboration, Search for dark matter in events with missing transverse momentum and a Higgs boson decaying into two photons in pp collisions at $\sqrt{s}=13 \mathrm{TeV}$ with the ATLAS detector, arXiv:2104.13240 [INSPIRE].

[20] J.M. Lindert, K. Kudashkin, K. Melnikov and C. Wever, Higgs bosons with large transverse momentum at the LHC, Phys. Lett. B 782 (2018) 210 [arXiv:1801.08226] [INSPIRE].

[21] J.C. Collins and D.E. Soper, Angular Distribution of Dileptons in High-Energy Hadron Collisions, Phys. Rev. D 16 (1977) 2219 [InSPIRE].

[22] ATLAS collaboration, Study of the spin and parity of the Higgs boson in diboson decays with the ATLAS detector, Eur. Phys. J. C 75 (2015) 476 [Erratum ibid. 76 (2016) 152] [arXiv: 1506.05669] [INSPIRE].

[23] K. Becker et al., Precise predictions for boosted Higgs production, arXiv:2005.07762 [INSPIRE].

[24] M. Czakon, A novel subtraction scheme for double-real radiation at NNLO, Phys. Lett. B 693 (2010) 259 [arXiv: 1005.0274] [INSPIRE].

[25] M. Czakon, Double-real radiation in hadronic top quark pair production as a proof of a certain concept, Nucl. Phys. B 849 (2011) 250 [arXiv:1101.0642] [InSPIRE].

[26] M. Czakon and D. Heymes, Four-dimensional formulation of the sector-improved residue subtraction scheme, Nucl. Phys. B 890 (2014) 152 [arXiv: 1408.2500] [InSPIRE].

[27] M. Czakon, P. Fiedler and A. Mitov, Resolving the Tevatron Top Quark Forward-Backward Asymmetry Puzzle: Fully Differential Next-to-Next-to-Leading-Order Calculation, Phys. Rev. Lett. 115 (2015) 052001 [arXiv: 1411.3007] [INSPIRE].

[28] M. Czakon, D. Heymes and A. Mitov, High-precision differential predictions for top-quark pairs at the LHC, Phys. Rev. Lett. 116 (2016) 082003 [arXiv: 1511.00549] [INSPIRE].

[29] M. Czakon, P. Fiedler, D. Heymes and A. Mitov, NNLO QCD predictions for fully-differential top-quark pair production at the Tevatron, JHEP 05 (2016) 034 [arXiv: 1601.05375] [INSPIRE].

[30] M. Czakon, D. Heymes and A. Mitov, Dynamical scales for multi-TeV top-pair production at the LHC, JHEP 04 (2017) 071 [arXiv:1606.03350] [INSPIRE].

[31] A. Behring, M. Czakon, A. Mitov, A.S. Papanastasiou and R. Poncelet, Higher order corrections to spin correlations in top quark pair production at the LHC, Phys. Rev. Lett. 123 (2019) 082001 [arXiv: 1901.05407] [INSPIRE].

[32] M. Czakon, A. van Hameren, A. Mitov and R. Poncelet, Single-jet inclusive rates with exact color at $\mathcal{O}\left(\alpha_{s}^{4}\right)$, JHEP 10 (2019) 262 [arXiv:1907.12911] [INSPIRE].

[33] M. Czakon, A. Mitov, M. Pellen and R. Poncelet, NNLO QCD predictions for $W+c$-jet production at the LHC, JHEP 06 (2021) 100 [arXiv:2011.01011] [INSPIRE]. 
[34] M.L. Czakon, T. Generet, A. Mitov and R. Poncelet, B-hadron hadro-production in NNLO QCD: application to LHC t $\bar{t}$ events with leptonic decays, arXiv:2102.08267 [INSPIRE].

[35] R. Poncelet and A. Popescu, NNLO QCD study of polarised $W^{+} W^{-}$production at the LHC, JHEP 07 (2021) 023 [arXiv:2102.13583] [INSPIRE].

[36] https://bitbucket.org/hameren/avhlib.

[37] M. Bury and A. van Hameren, Numerical evaluation of multi-gluon amplitudes for High Energy Factorization, Comput. Phys. Commun. 196 (2015) 592 [arXiv:1503.08612] [INSPIRE].

[38] F. Cascioli, P. Maierhofer and S. Pozzorini, Scattering Amplitudes with Open Loops, Phys. Rev. Lett. 108 (2012) 111601 [arXiv:1111.5206] [INSPIRE].

[39] F. Buccioni et al., OpenLoops 2, Eur. Phys. J. C 79 (2019) 866 [arXiv:1907.13071] [INSPIRE].

[40] H.A. Chawdhry, M. Czakon, A. Mitov and R. Poncelet, Two-loop leading-colour QCD helicity amplitudes for two-photon plus jet production at the LHC, JHEP 07 (2021) 164 [arXiv: 2103.04319] [INSPIRE].

[41] H.A. Chawdhry, M.A. Lim and A. Mitov, Two-loop five-point massless QCD amplitudes within the integration-by-parts approach, Phys. Rev. D 99 (2019) 076011 [arXiv: 1805.09182] [INSPIRE].

[42] D. Chicherin and V. Sotnikov, Pentagon Functions for Scattering of Five Massless Particles, JHEP 12 (2020) 167 [arXiv:2009.07803] [INSPIRE].

[43] H.A. Chawdhry, M. Czakon, A. Mitov and R. Poncelet, Two-loop leading-color helicity amplitudes for three-photon production at the LHC, JHEP 06 (2021) 150 [arXiv: 2012.13553] [INSPIRE].

[44] B. Agarwal, F. Buccioni, A. von Manteuffel and L. Tancredi, Two-loop leading colour QCD corrections to $q \bar{q} \rightarrow \gamma \gamma g$ and $q g \rightarrow \gamma \gamma q$, JHEP 04 (2021) 201 [arXiv:2102.01820] [INSPIRE].

[45] NNPDF collaboration, Parton distributions from high-precision collider data, Eur. Phys. J. $C \mathbf{7 7}(2017) 663$ [arXiv:1706.00428] [INSPIRE].

[46] A. Buckley et al., LHAPDF6: parton density access in the LHC precision era, Eur. Phys. J. $C 75$ (2015) 132 [arXiv:1412.7420] [InSPIRE].

[47] CMS collaboration, Search for supersymmetry using Higgs boson to diphoton decays at $\sqrt{s}=13 \mathrm{TeV}$, JHEP 11 (2019) 109 [arXiv:1908.08500] [INSPIRE].

[48] S. Frixione, Isolated photons in perturbative QCD, Phys. Lett. B 429 (1998) 369 [hep-ph/9801442] [INSPIRE].

[49] B. Agarwal, F. Buccioni, A. von Manteuffel and L. Tancredi, Two-loop helicity amplitudes for diphoton plus jet production in full color, arXiv:2105.04585 [INSPIRE]. 